\title{
Editorial: Resident and Ectopic FGF Signaling in Development and Disease
}

\author{
Fen Wang ${ }^{1,2 *}$, Xiaokun $L^{3}$ and Cong Wang ${ }^{3}$ \\ ${ }^{1}$ Texas A\&M Health Science Center, Institute of Biosciences and Technology, College Station, TX, United States, \\ ${ }^{2}$ Department of Translational Medicine, College of Medicine, Texas A\&M University, College Station, TX, United States, \\ ${ }^{3}$ School of Pharmaceutical Sciences, Wenzhou Medical University, Wenzhou, China
}

Keywords: fibroblast growth factor, receptor tyrosine kinase, intrinsic cell signaling, ectopic cell signaling, canonical signaling, non-canonical signaling

\section{Editorial on the Research Topic}

\section{Resident and Ectopic FGF Signaling in Development and Disease}

The fibroblast growth factor (FGF) family is one of the largest growth factor families, which consists of 18 receptor-binging, intrinsic tissue regulatory polypeptides that share similarity in structures and amino acid sequences (Mckeehan et al., 2009; Wang et al., 2013, 2019). The FGFs are broadly expressed, which regulate cell proliferation, differentiation, survival, and function. The FGFs elicit their regulatory activities by binding and activating the FGF receptor (FGFR) transmembrane tyrosine kinases encoded by four highly homologous genes. Beside the FGF and FGFR, the FGF signaling complex also includes highly diverse heparan sulfate (HS) polysaccharides and klothos as co-receptors that not only affect the ligand-binding activity, but also modulate signaling specificity of the FGFR. Binding of the FGF to the FGFR-HS complex changes the conformation of the complexes, leading to receptor autophosphorylation, as well as phosphorylation of downstream signaling molecules, and thus, transmits the signals to downstream effectors. Both FGF and FGFR are expressed in a highly spatiotemporally and cell type-specific manner. Ectopic expression of the FGF and FGFR isoforms has been identified as culprits for multiple diseases, including developmental defects, cancer, and metabolic disorders (Li et al., 2016; Wang et al., 2019).

As an intrinsic cell signaling axis, the FGF and FGFR are expressed almost ubiquitously in all tissues and stages, function through the autocrine, paracrine, or endocrine mechanisms. These intrinsic FGF signaling axes play important roles in embryonic development, adult tissue homeostasis, function, and regeneration. Redundant FGF and FGFR expression are common in multiple tissues and organs to warrant the signaling conveyance. Loss of FGF signaling has been reported as culprits for developmental disorders and many other diseases. However, when ectopically expressed, the FGF signaling axis is pathogenic in many cells and tissues, ranging from birth defects, metabolic disorders, to cancers and cardiovascular diseases. Multiple mechanisms have been attributed to ectopic FGF signaling, including ectopic expression of FGF, FGFR, and heparan sulfate proteoglycans (HSPG), gain-of-function mutations in the FGFR tyrosine kinases, and possibly other unidentified mechanisms.

Upon activation, FGFRs elicit both canonical and non-canonical signals. The canonical signals include FRS2 $\alpha$-dependent activation of ERK and PI3K, as well as FRS2 $\alpha$-independent activation of PLC $\gamma$ (Zhang et al., 2008a,b, 2010, 2012a,b; Lin et al., 2011; Li et al., 2016; Wang et al., 2016, 2019); non-canonical signaling pathways include posttranslational modifications of lactate dehydrogenase A (LDHA) and transforming growth factor $\beta$-activated kinase 1 (TAK1), which increases the stability, as well as the activity of these enzymes 
(Zhang et al., 2004; Lan et al., 2008; Fan et al., 2011; Jin et al., 2017; Liu et al., 2018; Wang et al., 2018). Although FGFR1-4 are highly similar, share many common downstream pathways, and elicit similar canonical signals in some cells, they elicit opposite signals in many other cell types (Feng et al., 1997; Jin et al., 2003; Wang et al., 2019). The mechanism underlying this signaling specificity is poorly understood. Converging data showed that non-canonical signaling likely accounts for the receptor isoform-specific signals. However, how the four FGFRs elicit receptor-isoform signals still largely remains to be elucidated. This is partly due to the complexity of the FGF signaling axis, which includes multiple ligands, receptors, and cofactors, as well as poorly understood non-canonical downstream pathways that mediate receptor isoform-specific signals (Mckeehan et al., 2009; Wang et al., 2013; Li et al., 2016). Understanding how FGFR elicits receptor isoform-specific signals will provide new strategies for precision medicine for diseases that are caused by loss of intrinsic FGF signaling or gain of ectopic FGF signaling.

This Research Topic includes several review articles of important concepts related to how intrinsic FGF signaling controls stem cell self-renewal and differentiation, organ development, repair, and regeneration, how dysregulated FGF19-FGFR4 signaling axis contributes to hepatocellular carcinoma, as well as several original studies that reveal novel aspects of how intrinsic FGF signaling alleviates inflammation responses in the kidney and liver, how ectopic FGF signaling promotes inflammation in the liver and increases stemness of cancer stem cells, and how engineered FGF2 has improved therapeutic values by reducing the undesirable heparin-binding activity.

Stem cells play an important role in regenerative medicine. Understand how to regulate stem cells to remain dormant, or activate them to undergo self-renewal and differentiation will provide new therapeutic strategies for regenerative medicine. Mossahebi-Mohammadi et al. systematically reviewed the expression and function of FGF signaling pathway in stem cells, induced pluripotent stem cells, and epiblast-derived stem cells, and how it controlled the pluripotency and differentiation of these stem cells. Furthermore, they also discussed the cross talks of the FGF pathways with other signaling pathways, including transforming growth factor $\beta$, Wnt, and retinoic acid, to control stem cell self-renewal and differentiation.

Cancer stem cells are culprits for relapse of malignant cancer. Both in vitro and in vivo experiments show that spheroid-forming cancer stem cells have high tumorigenic activity. Quan et al. reported that the FGF-FGFR signaling axis is required for maintaining spheroid-forming in vitro and tumor-forming in vivo of pancreatic ductal adenocarcinoma cells. They further demonstrated that the FGF signals in these stem cells are mediated by the AKT pathway, which prevent SOX2 degradation and increase SOX2 nuclear localization. Inhibition of AKT activity impaired sphere-forming activity in vitro and tumor-forming activity in vivo. The authors also discussed how suppression of this signaling axis was of therapeutic value for pancreatic cancer.

FGF signaling plays an important role in embryonic development and organogenesis. The rudimentary digestive tract is initially a tube-like structure that is composed of epithelial cells surrounded by mesenchymal cells, which develops into distinct functional regions: the tongue, the pharynx, the esophagus, the stomach, the duodenum, the small intestine, the cecum, the large intestine, the colon, and the anus, as well as the pancreas and the liver. Lv et al. systematically reviewed how FGF10-FGFR2 signaling axis regulated the development of the digestive tract, including the taste papillae, tongue, salivary gland, stomach, pancreas, and liver. They also discussed how FGFR2 signaling regulated intestinal injury repair and the translational use of recombinant FGF7 and FGF10 to improves the short bowl syndrome, alleviated inflammatory bowel disease and ulcerative colitis disease.

As an endocrine FGF, FGF19 secreted from small intestine activates FGFR4 in the hepatocytes and regulates bile acid production and secretion ( $\mathrm{Yu}$ et al., 2000, 2005; Wang et al., 2014). FGFR4 is an intrinsic FGFR in hepatocytes, it plays an important role in liver function and suppresses chemicalinduced inflammation and tumorigenesis (Yu et al., 2002, 2003; Zhao et al., 2006; Huang et al., 2007, 2009; Luo et al., 2010). However, ectopic expression and activation of the FGF19FGFR4 signaling axis is pathogenic. Liu et al. summarized the expression and signaling pathway of the FGF19-FGFR4 signaling axis, and how ectopic FGF19-FGFR4 signaling was associated with many types of cancer. Overactivated FGFR4 promoted proliferation and prevented apoptosis of hepatocellular carcinoma. Ectopically activation of FGFR4 was detected in breast cancer, head and neck squamous cell carcinoma, colorectal cancer and lung cancer. They also discussed how FGFR4 inhibition, either with small molecular inhibitor or neutralizing antibodies, could be used to treat cancer that overexpressed ectopic FGFR4.

Inflammation is a double-edged sword for the host. Overactive inflammation exacerbates injuries, including acute kidney injury and liver damage induced by concanavalin $\mathrm{A}$. Tan et al. reported that treating the mice with FGF2 activated the FGFR signaling pathway in kidney cells both in vitro and in vivo, and reduced inflammatory reactions, and thereby, alleviated kidney damage. Furthermore, they also unraveled that the canonical FGF downstream pathways, ERK and AKT, were required for FGF2 to alleviate inflammation in kidney cells. Wang et al. also reported that human hepatic stellate cells expressed FGF21, FGFR1, and beta-Klotho (KLB). The expression of this intrinsic FGF signaling axis likely cross talked with the TNF $\alpha$ pathway. Overexpression of FGF21 in mice alleviated toxin-induced inflammation and damages in the liver. However, treating hepatic stellate cells with FGF1 that bound to FGFR1 independent of KLB activated the non-canonical TAK1-mediated TNF $\alpha$ pathway and exacerbated inflammation reaction in the cells. The results demonstrate that the intrinsic FGF21-FGFR1/KLB signaling axis and ectopic FGF1-FGFR1 elicit functional opposite signals with respect to regulating inflammation.

Many members of the FGF family have the heparan sulfate/heparin binding activity (Li et al., 2016). As a co-factor, heparan sulfates enhance the binding of FGF and FGFR. They also determine the receptor-binding profiles of FGF, as well as signaling profile of the FGFR, and stability of the FGF. Koledova 
et al. tested two engineered FGF2 that have low heparinbinding activities with respect to stability, heparin dependence, and dynamics of activating ERK. They found that the two FGF2 derivatives, FGF2-STAB1 and FGF2-STAB2, had a higher mitogenic activity than did the wildtype FGF2. They also induced ERK phosphorylation more potently and at a faster dynamic than wildtype FGF2. Since the engineered FGF2s do not bind to heparin and have a high activity in activating the canonical ERK pathway, they are of transitional values for activating the FGFRERK signaling pathway in the precision medicine (Li et al., 2016).

In summary, the FGF signaling is complicated, and in many cases, the signaling specificity and intensity is not only FGF and FGFR isoform-dependent, but also heparan sulfate and host cells-dependent. It can be either a foe or friend, and the only determinant of what they are is when, where, and how the

\section{REFERENCES}

Fan, J., Hitosugi, T., Chung, T. W., Xie, J., Ge, Q., Gu, T. L., et al. (2011). Tyrosine phosphorylation of lactate dehydrogenase A is important for $\mathrm{NADH} / \mathrm{NAD}(+)$ redox homeostasis in cancer cells. Mol. Cell. Biol. 31, 4938-4950. doi: 10.1128/MCB.06120-11

Feng, S., Wang, F., Matsubara, A., Kan, M., and Mckeehan, W. L. (1997). Fibroblast growth factor receptor 2 limits and receptor 1 accelerates tumorigenicity of prostate epithelial cells. Cancer Res. 57, 5369-5378.

Huang, X., Yang, C., Jin, C., Luo, Y., Wang, F., and Mckeehan, W. L. (2009). Resident hepatocyte fibroblast growth factor receptor 4 limits hepatocarcinogenesis. Mol. Carcinog. 48, 553-562. doi: $10.1002 / \mathrm{mc}$. 20494

Huang, X., Yang, C., Luo, Y., Jin, C., Wang, F., and Mckeehan, W. L. (2007). FGFR4 prevents hyperlipidemia and insulin resistance but underlies high-fat diet induced fatty liver. Diabetes 56, 2501-2510. doi: 10.2337/ $\mathrm{db} 07-0648$

Jin, C., Mckeehan, K., Guo, W., Jauma, S., Ittmann, M. M., Foster, B., et al. (2003). Cooperation between ectopic FGFR1 and depression of FGFR2 in induction of prostatic intraepithelial neoplasia in the mouse prostate. Cancer Res. 63, 8784-8790.

Jin, L., Chun, J., Pan, C., Alesi, G. N., Li, D., Magliocca, K. R., et al. (2017). Phosphorylation-mediated activation of LDHA promotes cancer cell invasion and tumour metastasis. Oncogene 36, 3797-3806. doi: 10.1038/onc.2017.6

Lan, Y., Zhang, Y., Wang, J., Lin, C., Ittmann, M. M., and Wang, F. (2008). Aberrant expression of $\mathrm{Cks} 1$ and $\mathrm{Cks} 2$ contributes to prostate tumorigenesis by promoting proliferation and inhibiting programmed cell death. Int. J. Cancer 123, 543-551. doi: 10.1002/ijc.23548

Li, X., Wang, C., Xiao, J., Mckeehan, W. L., and Wang, F. (2016). Fibroblast growth factors, old kids on the new block. Semin Cell Dev Biol. 53, 155-167. doi: $10.1016 / j . s e m c d b .2015 .12 .014$

Lin, X., Zhang, Y., Liu, L., Mckeehan, W. L., Shen, Y., Song, S., et al. (2011). FRS2alpha is essential for the fibroblast growth factor to regulate the mTOR pathway and autophagy in mouse embryonic fibroblasts. Int. J. Biol. Sci. 7, 1114-1121. doi: 10.7150/ijbs.7.1114

Liu, J., Chen, G., Liu, Z., Liu, S., Cai, Z., You, P., et al. (2018). Aberrant FGFR tyrosine kinase signaling enhances the warburg effect by reprogramming LDH isoform expression and activity in prostate cancer. Cancer Res. 78, 4459-4470. doi: 10.1158/0008-5472.CAN-17-3226

Luo, Y., Yang, C., Lu, W., Xie, R., Jin, C., Huang, P., et al. (2010). Metabolic regulator betaKlotho interacts with fibroblast growth factor receptor 4 (FGFR4) to induce apoptosis and inhibit tumor cell proliferation. J. Biol. Chem. 285, 30069-30078. doi: 10.1074/jbc.M110.148288

Mckeehan, W. L., Wang, F., and Luo, Y. (2009). "The fibroblast growth factor (FGF) signaling complex," in Handbook of Cell Signaling eds signals are elicited. Yet, how resident FGF-FGFR signaling axis elicits canonical or non-canonical signals to regulate multiple cellular activities and how ectopic FGF signaling causes diseases largely remain an enigma. Solving this enigma is needed for developing FGF-based precision therapies to treat diseases caused by aberrant FGF signals.

\section{AUTHOR CONTRIBUTIONS}

FW, XL, and CW conceived and wrote the editorial. All authors contributed to the article and approved the submitted version.

\section{FUNDING}

The work is partially supported by CPRIT to FW (RP190612).
R. Bradshaw and E. Dennis (New York, NY: Academic/Elsevier Press). doi: 10.1016/B978-0-12-374145-5.00038-3

Wang, C., Ke, Y., Liu, S., Pan, S., Liu, Z., Zhang, H., et al. (2018). Ectopic fibroblast growth factor receptor 1 promotes inflammation by promoting nuclear factor-kappaB signaling in prostate cancer cells. J. Biol. Chem. 293, 14839-14849. doi: 10.1074/jbc.RA118.002907

Wang, C., Liu, Z., Ke, Y., and Wang, F. (2019). Intrinsic FGFR2 and ectopic FGFR1 signaling in the prostate and prostate cancer. Front. Genet. 10:12. doi: $10.3389 /$ fgene.2019.00012

Wang, C., McKeehan, W. L., and Wang, F. (2016). "FRS2a At the center of FGF signaling," in Fibroblast Growth Factors, ed M. Simons (Hackensack, NJ World Scientific), 73-110. doi: 10.1142/9789813143371_0003

Wang, C., Yang, C., Chang, J. Y., You, P., Li, Y., Jin, C., et al. (2014) Hepatocyte FRS2alpha is essential for the endocrine fibroblast growth factor to limit the amplitude of bile acid production induced by prandial activity. Curr. Mol. Med. 14, 703-711. doi: 10.2174/15665240146661407240 95112

Wang, F., Luo, Y., and Mckeehan, W. (2013). "The FGF signaling axis in prostate tumorigenesis," in Molecular Oncology: Causes of Cancer and Targets for Treatment (Cambridge: London Cambridge University Press), 186-189. doi: 10.1017/CBO9781139046947.017

Yu, C., Wang, F., Jin, C., Huang, X., and Mckeehan, W. L. (2005). Independent repression of bile acid synthesis and activation of c-Jun $\mathrm{N}$-terminal kinase (JNK) by activated hepatocyte fibroblast growth factor receptor 4 (FGFR4) and bile acids. J. Biol. Chem. 280, 17707-17714. doi: 10.1074/jbc.M411771200

Yu, C., Wang, F., Jin, C., Huang, X., Miller, D. L., Basilico, C., et al. (2003). Role of fibroblast growth factor type 1 and 2 in carbon tetrachlorideinduced hepatic injury and fibrogenesis. Am. J. Pathol. 163, 1653-1662. doi: 10.1016/S0002-9440(10)63522-5

Yu, C., Wang, F., Jin, C., Wu, X., Chan, W. K., and Mckeehan, W. L. (2002). Increased carbon tetrachloride-induced liver injury and fibrosis in FGFR4-deficient mice. Am. J. Pathol. 161, 2003-2010. doi: 10.1016/S0002-9440(10)64478-1

Yu, C., Wang, F., Kan, M., Jin, C., Jones, R. B., Weinstein, M., et al. (2000). Elevated cholesterol metabolism and bile acid synthesis in mice lacking membrane tyrosine kinase receptor FGFR4. J. Biol. Chem. 275, 15482-15489. doi: $10.1074 /$ jbc.275.20.15482

Zhang, J., Chang, J. Y., Huang, Y., Lin, X., Luo, Y., Schwartz, R. J., et al. (2010). The FGF-BMP signaling axis regulates outflow tract valve primordium formation by promoting cushion neural crest cell differentiation. Circ. Res. 107, 1209-1219. doi: 10.1161/CIRCRESAHA.110.225318

Zhang, J., Lin, Y., Zhang, Y., Lan, Y., Lin, C., Moon, A. M., et al. (2008a). Frs2alpha-deficiency in cardiac progenitors disrupts a subset of FGF signals required for outflow tract morphogenesis. Development 135, 3611-3622. doi: $10.1242 /$ dev. 025361 
Zhang, J., Liu, J., Huang, Y., Chang, J. Y., Liu, L., Mckeehan, W. L., et al. (2012a). FRS2alpha-mediated FGF signals suppress premature differentiation of cardiac stem cells through regulating autophagy activity. Circ. Res. 110, e29-e39. doi: 10.1161/CIRCRESAHA.111.255950

Zhang, J., Liu, J., Liu, L., Mckeehan, W. L., and Wang, F. (2012b). The fibroblast growth factor signaling axis controls cardiac stem cell differentiation through regulating autophagy. Autophagy 8, 690-691. doi: 10.4161/auto.19290

Zhang, Y., Lin, Y., Bowles, C., and Wang, F. (2004). Direct cell cycle regulation by the fibroblast growth factor receptor (FGFR) kinase through phosphorylationdependent release of Cks1 from FGFR substrate 2. J. Biol. Chem. 279, 55348-55354. doi: 10.1074/jbc.M409230200

Zhang, Y., Zhang, J., Lin, Y., Lan, Y., Lin, C., Xuan, J. W., et al. (2008b). Role of epithelial cell fibroblast growth factor receptor substrate 2 alpha\} in prostate development, regeneration and tumorigenesis. Development 135, 775-784. doi: 10.1242/dev.009910
Zhao, P., Caretti, G., Mitchell, S., Mckeehan, W. L., Boskey, A. L., Pachman, L. M., et al. (2006). Fgfr4 is required for effective muscle regeneration in vivo. Delineation of a MyoD-Tead2-Fgfr4 transcriptional pathway. J. Biol. Chem. 281, 429-438. doi: 10.1074/jbc.M507440200

Conflict of Interest: The authors declare that the research was conducted in the absence of any commercial or financial relationships that could be construed as a potential conflict of interest.

Copyright (c) 2020 Wang, Li and Wang. This is an open-access article distributed under the terms of the Creative Commons Attribution License (CC BY). The use, distribution or reproduction in other forums is permitted, provided the original author(s) and the copyright owner(s) are credited and that the original publication in this journal is cited, in accordance with accepted academic practice. No use, distribution or reproduction is permitted which does not comply with these terms. 